\title{
Comparison of SOX-10, HMB-45, and Melan-A in Benign Melanocytic Lesions
}

\author{
Sabrina E Dass' \\ Taryn Huizenga ${ }^{2}$ \\ Mehdi Farshchian (iD) ${ }^{2}$ \\ Darius R Mehregan ${ }^{2}$ \\ 'Wayne State University School of \\ Medicine, Detroit, MI, USA; ${ }^{2}$ Department \\ of Dermatology, Wayne State University, \\ Detroit, MI, USA
}

Purpose: Different immunohistochemical stains are used in dermatopathology to stain melanocytes and diagnose benign and malignant melanocytic lesions.

Methods: SOX-10, HMB-45, and Melan-A immunohistochemical stains were used to assess 32 biopsy specimens with a histologic diagnosis of lentigo. The total number of melanocytes stained with each immunohistochemical stain was counted and an average count was obtained from two readings.

Results: Analysis of the data revealed a significant difference in staining melanocytes between these three immunostains ( $\mathrm{p}=0.0010$, ANOVA). SOX-10 stained 0.195 more melanocytes than HMB-45 ( $\mathrm{p}=0.0026$ ). Similarly, Melan-A stained 0.195 more melanocytes than HMB-45 ( $\mathrm{p}=0.0011)$. However, the difference between SOX-10 and Melan-A was not statistically significant $(\mathrm{p}=0.9810)$.

Conclusion: SOX-10 and Melan-A immunostaining stain more melanocytes than HMB-45. No significant difference was noted between Melan-A and SOX-10.

Keywords: immunohistochemical stains, melanocytes, benign melanocytic lesion, lentigo, atypical junctional melanocytic hyperplasia

\section{Introduction}

Early melanoma in situ can be difficult to distinguish from lentigo senilis, particularly in sun-damaged skin, which can result in a significant delay in diagnosis. ${ }^{1,2}$ Efforts to identify additional prognostic biomarkers in melanocytic lesions continue to be a challenge. Hematoxylin and eosin (H\&E) staining in conjunction with immunohistochemistry has remained the gold standard for dermatopathologists to confirm their diagnoses. ${ }^{3}$ The choice of immunohistochemical stains may depend on the pathologists' personal preferences; however, data on comparison between different immunostains are scarce.

Widely used immunohistochemical markers for the diagnosis of melanocytic lesions include Sry-related HMG-BOX gene 10 (SOX-10), human melanoma black-45 (HMB-45), and Melan-A/MART-1. These three immunohistochemical stains are used to visualize melanocytes in melanocytic lesions expressed in the epidermis and dermo-epidermal junction. SOX-10 is a nuclear transcription factor involved in the differentiation of neural crest cells to melanocytes. ${ }^{2}$ Its nuclear pattern differentiates itself from the other commonly used melanocytic markers. It has been shown to be both a sensitive and specific marker in staining melanomas. ${ }^{4}$ HMB-45 is a monoclonal cytoplasmic marker of the premelanosome complex, glycoprotein gp100. The HMB-45 stain is less sensitive, but fairly specific for
Correspondence: Mehdi Farshchian Department of Dermatology, Wayne State University, I8I00 Oakwood Blvd. Suite 300, Detroit, MI, 48I24, USA Email mfarshch@med.wayne.edu 
melanocytic differentiation. ${ }^{5}$ Melan-A/MART-1, also known as melanoma antigen, recognized by $\mathrm{T}$ cells, is a cytoplasmic protein. ${ }^{6}$ In addition to staining melanocytes, Melan-A has been reported to stain melanophages. The Melan-A immunostain has an equal sensitivity and specificity for melanoma; however, it has been shown to stain melanoma more intensely and may appear to show more epidermal melanocytes than what is actually present. ${ }^{3}$ Since there has not been a single antigen identified that stains with maximum sensitivity and specificity, immunohistochemical markers are typically used in combination.

Although immunohistochemistry has been the gold standard to distinguish melanocytic lesions, including benign versus malignant, histological diagnosis can be difficult, which has led to an increasing interest in finding a marker that is most beneficial in staining melanocytes. To date, there are no studies comparing the staining patterns of SOX-10, HMB-45, and Melan-A of melanocytes in lentigo senilis or benign melanocytic lesions. The objective of our study is to compare SOX-10, HMB-45, and Melan-A to determine the relative degree of immunohistochemical staining in lentigo senilis.

\section{Methods}

Thirty-two biopsy specimens with a histologic diagnosis of lentigo were randomly selected from the database at Pinkus Dermatopathology Lab, Monroe, MI. Lesions were chosen based on a clinical suspicion of lentigo maligna (melanoma in situ) but were histologically lentigo senilis or lentigo with focal melanocytic hyperplasia.

Each tissue section was formalin fixed (Thermo Scientific, Waltham, MA), embedded in paraffin (Cardinal Health, Dublin, $\mathrm{OH}$ ), and cut at $4 \mu \mathrm{m}$ thick. They were mounted onto charged slides and placed in a drying oven for 45 minutes at $60^{\circ} \mathrm{C}$. The sections were immunostained with anti-SOX-10 antibody (prediluted RTU, Cell Marque, Rocklin, CA), anti-HMB-45 antibody (pre-diluted RTU, Leica Biosystems, Buffalo Grove, IL), and anti-Melan-A antibody (pre-diluted RTU, Leica Biosystems, Buffalo Grove, IL), using an automated Leica Bond-Max Polymer Refine Detection Kit (Leica Biosystems, Buffalo Grove, IL). The total number of melanocytes stained with each immunohistochemical stain was counted by two independent observers (DRM and TH). An average count was obtained from the two independent readings.
ANOVA test was used to analyze the significance of difference between three immunohistochemical stains. A linear mixed effects model was also employed to test the difference in the count of melanocytes. A p-value less than 0.05 was considered statistically significant.

\section{Results}

The study included a total of 32 benign melanocytic lesions from 32 biopsy specimens with lentigo senilis or mild junctional proliferation (Table 1). In all of the cases, the melanocytes stained positive for SOX-10, HMB-45, and Melan-A. Specimens stained more intensely with Melan-A and SOX-10 at the dermo-epidermal junction compared to HMB-45; however, the difference between SOX-10 and Melan-A was not statistically significant $(p=0.9810$, Figures 1 and 2). The difference in the number of stained melanocytes was highly significant between HMB-45 and SOX-10 ( $\mathrm{p}=0.0031)$ and between HMB-45 and Melan-A ( $\mathrm{p}=0.0042)$ based on a pairwise comparison. Using a logarithmic scale, SOX-10 stained 0.195 more melanocytes $/ \mathrm{mm}$, or 1.215 times more, than HMB-45 $(p=0.0026)$. Similarly, Melan-A stained 0.195 more melanocytes $/ \mathrm{mm}$, or 1.214 times more, than HMB-45 $(p=0.0011$, Figure 3). Overall, the three markers have a significantly different ability to stain melanocytes $(p=0.0010$, ANOVA $)$.

\section{Discussion}

Immunohistochemistry remains the most common aid used by dermatopathologists to assist in the distinction between benign and malignant melanocytic lesions. The S100 marker has historically been used as a melanocytic marker; however, it is highly sensitive but not specific for melanocytes as it is also expressed in epidermal Langerhans cells. Unfortunately, there still is not a single immunohistochemical marker that can achieve this distinction with certainty. $^{7}$ Often a subtle melanocytic proliferation can be easily missed. In particular, an early melanoma in situ may be difficult to differentiate from a lentigo senilis. Often this may lead to descriptive diagnoses such as "lentigo with junctional melanocytic hyperplasia" or "atypical junctional melanocytic proliferation". All of the cases studied were submitted to rule out melanoma in situ but showed histologic findings of a lentigo senilis or mild atypical junctional proliferation. No cases showed definitive features of a melanoma such as pagetoid scatter of melanocytes or melanocytic nest formation. SOX-10, HMB-45, and Melan-A are highly specific for melanocytic 


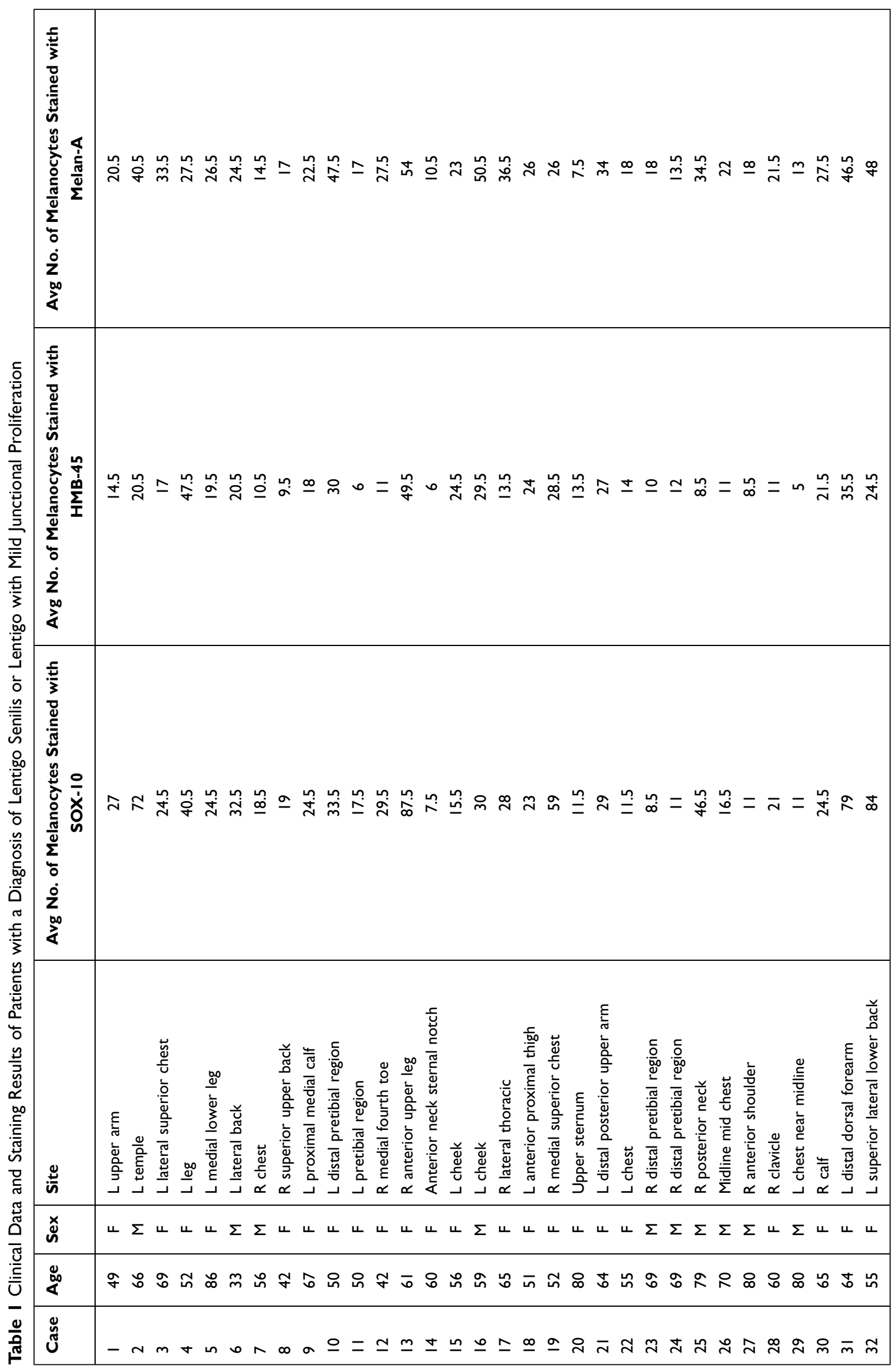



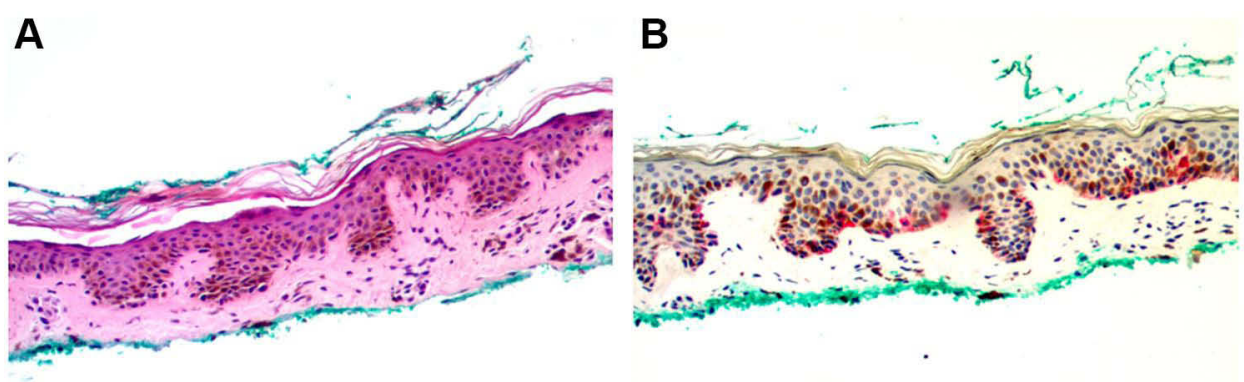

C

D
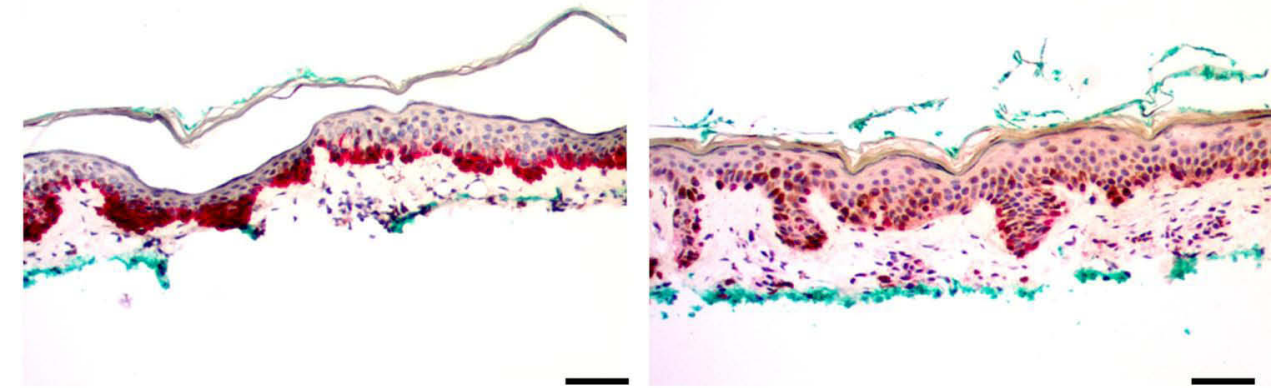

Figure I Mild atypical junctional proliferation. (A) H\&E stain, (B) HMB-45 stain, (C) Melan-A stain, (D) SOX-10 stain. More intense staining noted for Melan-A and SOX-10 at the dermo-epidermal junction. Scale bar $=50 \mu \mathrm{m}$.

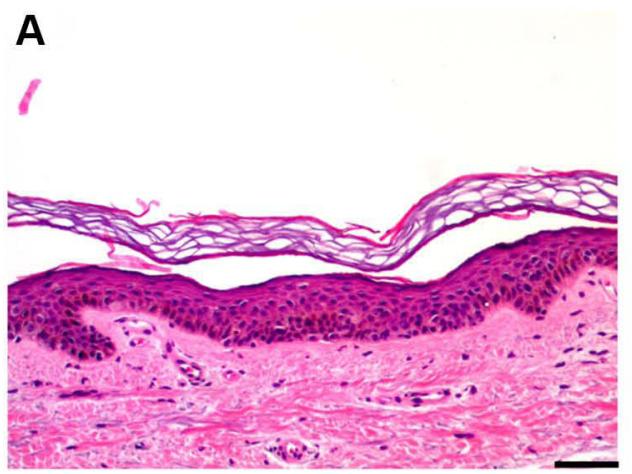

C

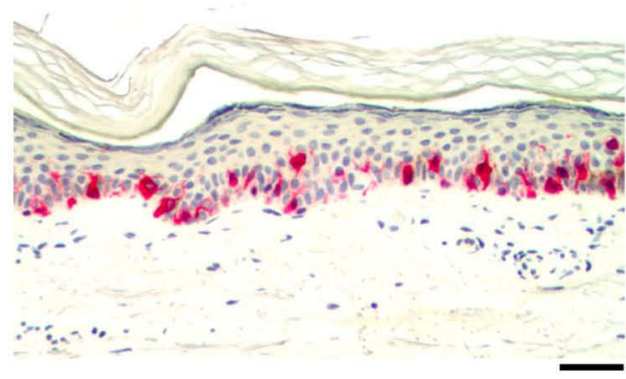

B

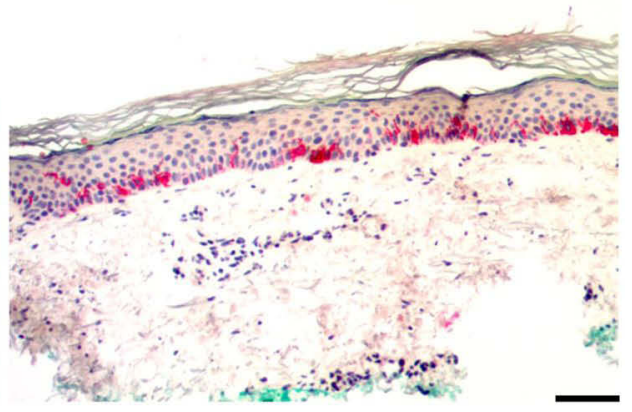

D

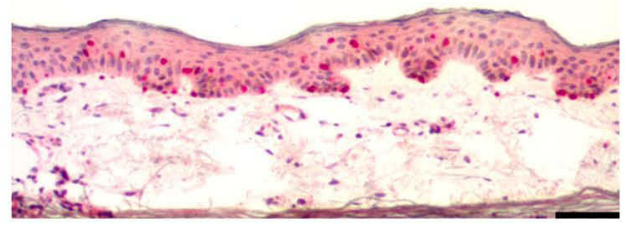

Figure 2 Lentigo with focal junctional melanocytic hyperplasia. (A) H\&E stain, (B) HMB-45 stain, (C) Melan-A stain, (D) SOX-10 stain. Melan-A and SOX-10 showed increased staining at the dermo-epidermal junction. Scale bar $=50 \mu \mathrm{m}$. 


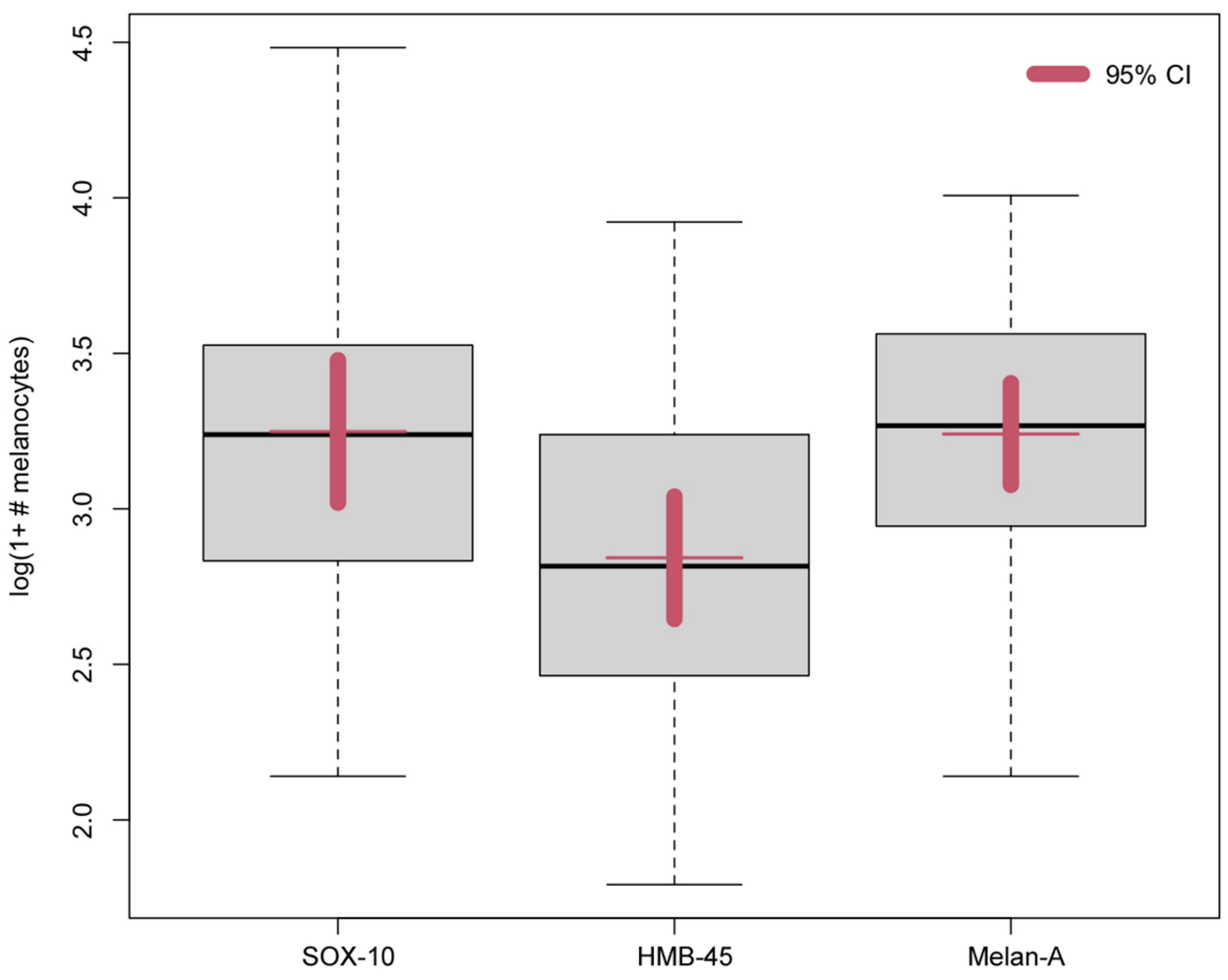

Figure 3 In a logarithmic scale, SOX-10 stained I.2I5 times more melanocytes than HMB-45 ( $\mathrm{p}=0.0026)$ and Melan-A stained I.2I4 times more melanocytes than HMB-45 $(p=0.001$ I) with a $95 \%$ confidence interval.

cell types. However, our findings show that the choice of immunohistochemical stain may result in different sensitivity for melanocytes. In our study, Melan-A and SOX-10 both had a significant increase in staining compared with HMB-45, however, there was no significant difference in staining between Melan-A and SOX-10. As in Figure 1, there was a prominent difference in staining of melanocyte dendrites between Melan-A and SOX-10. Even though there was not a statistical difference in the number of melanocytes stained, it was easier for the investigators to visualize melanocytes stained with SOX-10 compared to Melan-A. This is most obvious in Figure 1, however also visible in Figure 2.

HMB-45 and Melan-A have been previously compared in both benign and malignant melanocytic lesions. ${ }^{8,9}$ HMB-45 was found to be more specific, with greater staining at the dermo-epidermal junction, but lacking sensitivity. Moreover, Melan-A showed a diffuse staining of melanocytes in benign melanocytic lesions, being more sensitive overall. Melan-A was also found to stain heavily melanized keratinocytes and melanophages. This finding is consistent with what was seen in our stained biopsy specimens. Although HMB-45 did not stain as many melanocytes as SOX-10 and Melan-A, HMB-45 may be helpful in distinguishing between lentigo senilis and early melanoma in situ. This has been successfully demonstrated with staining of HMB-45 to differentiate pigmented actinic keratosis from lentigo maligna due to the reaction of HMB-45 in lesions with cytologic atypia and confluence of growth. ${ }^{10}$

A more recent study directly compared Melan-A and SOX-10 immunostaining. ${ }^{3}$ It was found that Melan-A stained significantly more melanocytes than SOX-10 in actinic keratoses; however, there was no difference in detecting melanocytic proliferations. Their results also showed that SOX-10 was more specific than Melan-A in identifying epidermal melanocytes on sun-damaged skin. ${ }^{4}$ In our study, we did not find a difference between MelanA and SOX-10 staining. Some authors argue that melanocytic cells can easily be overlooked in H\&E-stained sections and Melan-A should be the primary biomarker. With this in mind, Drabeni et al stained with Melan-A after initially staining with H\&E. Their findings resulted in $33 \%$ of in situ melanoma cases being reclassified as invasive melanoma. ${ }^{11}$ In an opposing study, they found an increased number of melanocytes in the epidermis confirmed by a strong positivity for Melan-A in single cells and in small nests located at the dermo-epidermal junction. 
However, their histopathology showed the presence of a lichenoid dermatitis, not melanoma in situ. These results suggest that immunohistochemical investigations performed with Melan-A alone may lead to confusing and potentially disastrous results in analyzing pigmented lesions. $^{12}$

This is particularly important for dermatologic surgeons and pathologists in distinguishing the subtle features of melanoma in situ in the background of atypical junctional melanocytic hyperplasia. Due to overlapping features of sun-damaged skin, reading the surgical margins after an excision can be challenging as there is often melanocytic hyperplasia with or without an associated pigmented lesion and variability in melanocyte density adjacent to melanoma and nonmelanoma skin cancers. ${ }^{13}$ Although melanocytic hyperplasia can be expected surrounding a melanoma on chronically sun-damaged skin, the progression of the melanocytic hyperplasia is still unclear. $^{14}$ Histologic discrimination of early melanoma in situ from benign melanocytic lesions can be challenging. Immunohistochemical studies of microphthalmiaassociated transcription factor (MITF), a key component of the signal transduction pathway for the development of and differentiation of melanocytes, showed increased melanocyte density and nuclear diameter in melanoma in situ compared to a benign solar lentigo. ${ }^{15,16}$ This demonstrates the importance in using a stain with a high specificity for melanocytes. The lack of specificity of Melan-A immunostaining, as stated previously, may result in falsepositive margins for surgical excision of melanoma in situ. In our study, Melan-A did not stain with clear, defined margins, possibly yielding more stained melanocytes than what was actually present. In addition, some authors have proposed that there is a continuum between solar lentigo and lentigo maligna. They have described it as a solar lentigo with melanocytic hyperplasia not extending past the margin of the lesion. Although distinguishing criteria for the diagnosis of melanoma in situ compared to melanocytic hyperplasia has been identified, there is uncertainty as to whether or not a solar lentigo may be a precursor lesion to lentigo maligna. ${ }^{17}$ This highlights the important role immunohistochemistry plays in the histopathological identification of benign melanocytic lesions from early malignancy.

Overall, we found a significant increase in staining with Melan-A and SOX-10 at the dermo-epidermal junction, compared to HMB-45. However, no significant difference was noted between Melan-A and SOX-10.
A potential limitation to our study is the limited sample size. Although a difference in melanocyte count was not observed between Melan-A and SOX-10, our results show an apparent trend approaching significance. Melan-A or SOX-10 may be superior in staining melanocytes in melanocytic lesions, however SOX-10 may be more specific. Immunohistochemistry itself carries a few obstacles including its small biopsy size with a limited number of melanocytes and its differing sensitivity and specificity of antibodies to differentiate between melanocytes and keratinocytes. ${ }^{12}$ Pathologists need to consider the relative sensitivity of the stains utilized when interpreting results of staining in subtle melanocytic proliferations.

In conclusion, immunohistochemical stains are commonly used to quantify melanocytic proliferation, especially in the differential diagnosis of lentigo senilis and early melanoma in situ. Our study shows that the choice of immunohistochemical stain may result in different sensitivity for melanocytic proliferation.

\section{Statement of Ethics}

This study was performed in accordance with ethical guidelines of the Declaration of Helsinki. Ethical approval to conduct this study was obtained from the Institutional Review Board (IRB) of Wayne State University, Detroit, MI. Consent requirement was waived as unidentified archived pathology materials were used in this study.

\section{Funding}

There is no funding to report.

\section{Disclosure}

The authors report no conflicts of interest in this work.

\section{References}

1. Okamura JM, Barr RJ, Cantos KA, et al. Benign atypical junctional melanocytic hyperplasia associated with intradermal nevi: a common finding that may be confused with melanoma in situ. Modern Pathol. 2000;13(8):857-860. doi:10.1038/modpathol.3880152

2. Muzumdar S, Argraves M, Kristjansson A, et al. A Quantitative comparison between SOX10 and MART-1 immunostaining to detect melanocytic hyperplasia in chronically sun-damaged skin. J Cutan Pathol. 2018;45(4):263-268. doi:10.1111/cup.13115

3. Kasprzak JM, Yaohui GX. Diagnosis and management of lentigo maligna: a review. Drugs Context. 2015;4:212281. doi:10.7573/ dic. 212281

4. Prieto VG, Shea RC. Immunohistochemistry of melanocytic proliferations. Arch Pathol Lab Med. 2011;135:7. doi:10.5858/20090717-RAR.1

5. Ohsie SJ, Sarantopoulos GP, Cochran AJ, et al. Immunohistochemical characteristics of melanoma. J Cutan Pathol. 2008;35(5):433-444. doi:10.1111/j.1600-0560.2007.00891.x 
6. Shabrawi-Caelen LE, Kerl H, Cerroni L. Melan-A: not a helpful marker in distinction between melanoma in situ on sun-damaged skin and pigmented actinic keratosis. Am J Dermatopathol. 2004;26 (5):364-366. doi:10.1097/00000372-200410000-00003

7. Koh SS, Cassarino DS. Immunohistochemical expression of p16 in melanocytic lesions: an updated review and meta-analysis. Arch Pathol Lab Med. 2018;142(7):815-828. doi:10.5858/arpa.2017-0435-RA

8. Willis BC, Johnson G, Wang J, et al. SOX10: a Useful marker for identifying metastatic melanoma in sentinel lymph nodes. App Immunohistochem Mol Morphol. 2015;23(2):109-112. doi:10.1097/ PAI.0000000000000097

9. Clarkson K, Sturdgess I, Molyneux A. The usefulness of tyrosinase in the immunohistochemical assessment of melanocytic lesions: a comparison of the novel T311 antibody (anti-tyrosinase) with S-100, HMB45, and A103 (anti-Melan-A). J Clin Pathol. 2001;54 (3):196-200. doi:10.1136/jcp.54.3.196

10. Ribé A, McNutt NS. S100A protein expression in the distinction between lentigo maligna and pigmented actinic keratosis. Am $J$ Dermatopathol. 2003;25(2):93-99. doi:10.1097/00000372200304000-00001

11. Drabeni M, Lopez-Vilaró L, Barranco C, et al. Differences in tumor thickness between hematoxylin and eosin and Melan-A immunohistochemically stained primary cutaneous melanomas. Am J Dermatopathol. 2013;35(1):56-63. doi:10.1097/ DAD.0b013e31825ba933
12. Beltraminelli H, Shabrawi-Caelen LE, Kerl H, et al. Melan-a-positive "pseudomelanocytic nests": a pitfall in the histopathologic and immunohistochemical diagnosis of pigmented lesions on sun-damaged skin. Am J Dermatopathol. 2009;31(3):305-308. doi:10.1097/ DAD.0b013e31819d3769

13. Barlow J, Maize J, Lang P. The density and distribution of melanocytes adjacent to melanoma and nonmelanoma skin cancers. Dermatologic Surg. 2007;33(2):199-207.

14. Johnson TM, Smith JW, Nelson BR, et al. Current therapy for cutaneous melanoma. J Am Acad Dermatol. 1995;32:689-707. doi:10.1016/0190-9622(95)91443-9

15. Bertolotto C, Abbe P, Hemesath TJ, et al. Microphthalmia gene product as a signal transducer in cAMP-induced differentiation of melanocytes. J Cell Biol. 1998;142:827-835. doi:10.1083/ jcb.142.3.827

16. Black WH, Thareja SK, Blake BP, et al. Distinction of melanoma in situ from solar lentigo on sun-damaged skin using morphometrics and MITF immunohistochemistry. Am J Dermatopathol. 2011;33 (6):573-578. doi:10.1097/DAD.0b013e3182093b13

17. Byrom L, Barksdale S, Weedon D, et al. Unstable solar lentigo: a defined separate entity. Australas $J$ Dermatol. 2016;57 (3):229-234. doi:10.1111/ajd.12447

\section{Publish your work in this journal}

Clinical, Cosmetic and Investigational Dermatology is an international, peer-reviewed, open access, online journal that focuses on the latest clinical and experimental research in all aspects of skin disease and cosmetic interventions. This journal is indexed on CAS.
The manuscript management system is completely online and includes a very quick and fair peer-review system, which is all easy to use. Visit http://www.dovepress.com/testimonials.php to read real quotes from published authors. 